\title{
Arteriovenous Malformation in CLOVES Syndrome
}

\author{
Ahmed Abu-Haniyeh MD ${ }^{1 *}$, Laith Alkukhun MD1, Mohammed Al-Natour MD², Muhammad Hassan ${ }^{3}$ and Adriano Tonelli \\ ${ }^{1}$ Internal Medicine Residency Program, Medicine Institute, Cleveland Clinic, Cleveland, USA \\ 2Interventional Radiology Department, Imaging Institute, Cleveland Clinic, Cleveland, USA \\ ${ }^{3}$ Department of Pulmonary, Allergy and Critical Care Medicine, Respiratory Institute, Cleveland Clinic, OH, USA \\ ${ }^{4}$ Jinnah Sindh Medical University, Karachi, Pakistan
}

\begin{abstract}
CLOVES syndrome (Congenital Lipomatous Overgrowth, Vascular malformations, and Epidermal nevi) is a rare and recently discovered syndrome. It is associated with vascular malformations and abnormal fatty tissue and skeletal growth. In this case, we report the clinical manifestations overtime in a patient with CLOVES syndrome who presented a unique arteriovenous malformation that led to severe neurological impairment by compressing the spinal cord. We also discuss the visceral findings in our patient, such as double ureter and splenomegaly, and describe the finding of corneal hydrops in both eyes and Chiari I malformation on brain imaging.
\end{abstract}

Keywords: CLOVES syndrome; Arteriovenous malformation; Disseminated intravascular coagulation

\section{Introduction}

CLOVES is a recently discovered syndrome [1-4]. This congenital (C) disease is linked to a somatic activating mutation in the PIK3CA gene [4] that causes asymmetrical lipomatous overgrowth (LO), vascular malformations $(\mathrm{V})$ and epidermal nevi (E) [1,2]. The acronym was later expanded to emphasize spinal and skeletal abnormalities, i.e. scoliosis and limbs length discrepancy; as well as seizures (S) $[5,6]$. Before the description of CLOVES syndrome, patients were misdiagnosed with overgrowth syndromes like Klippel-Trenaunay [3]. After the introduction of CLOVES syndrome, several case reports have been published [5,7-11]. The phenotypic manifestations of this disorder are evident at the time of birth. These manifestations progress with time leading to severe disability and life threatening complications [12-15]. For instance, lipomatous overgrowth in the lower extremities results in slaying of the soles and loss of function $[13,15]$. Eventually, necrosis induced by vascular entrapments leads to amputation [1315]. Furthermore, arteriovenous malformations (AVM) increase the risk of pulmonary embolism $[16,17]$ and can result in life threatening gastrointestinal bleeding [12]. They also commonly appear in the paraspinal region potentially leading to spinal cord compression $[12,15]$. Although there are multiple case reports of CLOVES syndrome, the gravity of its complications is under-recognized. We report a case of CLOVES syndrome with a detailed phenotypic description since the time of birth until the age of 26 years when he presented at our institution. One of the characteristics that stands out in this patient is the considerable size and unusual complexity of his AVM.

\section{Case Report}

This is a single case report. Hence, the approval of the ethics committee at our institution was waived. All images were released after obtaining informed consent from the patient's guardian. Our male patient was born at full term to a 21 year old G0P1 mother. Delivery was through caesarian section due to macrosomia. Birth weight was $4,980 \mathrm{~g}\left(>99^{\text {th }}\right.$ percentile) and length was $54 \mathrm{~cm}$ ( $>99^{\text {th }}$ percentile). APGAR scores were 7 at 1 minute and 9 at 5 minutes. Laboratory tests were normal. The mother did not have any significant past medical or surgical history. She was not exposed to toxins, medications, alcohol or smoking during pregnancy. She had usual prenatal care and all laboratory tests during pregnancy were apparently normal. At birth, patient had elongated face, fatty tissue hypertrophy of his lower limbs with length discrepancy, triangular feet and wide first interdigital space. Multiple port wine stains were noted on his chest, face and arms (Figure 1). He was initially diagnosed as Klippel-Trenaunay syndrome. During the first few years of life, he developed kyphosis, severe lymphedema and worsening of the fatty tissue hypertrophy of the lower limbs (Figure 2). He also suffered from developmental delay. He sat at 8 months, walked at 20 months and could only speak a few words at the age of 5 . He had a neuropsychological evaluation at age 17 that showed an intellectual quotient of 36 . He has two healthy younger siblings. At the age of 10 years, he had an upper gastrointestinal bleeding. Esophagogastroduodenoscopy revealed an AVM in the stomach and therefore underwent partial gastrectomy. At age of 13, he developed right corneal hydrops that evolved with significant corneal scarring and worsening vision requiring corneal transplant on the right eye. He developed left corneal hydrops at the age of 14 and underwent

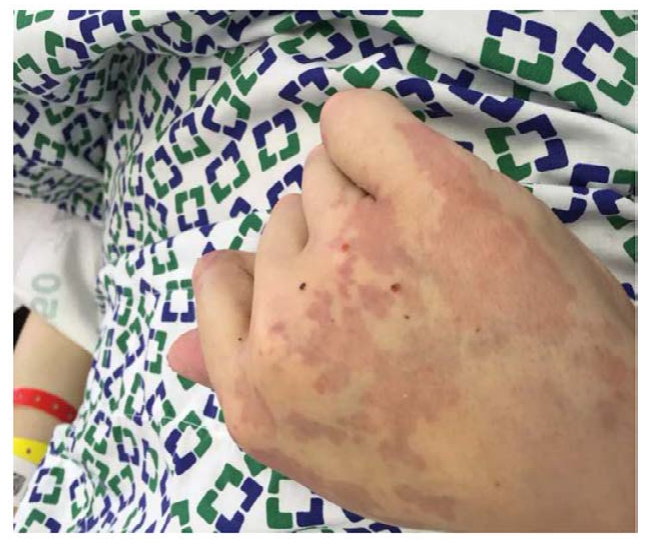

Figure 1: Port wine stain

*Corresponding author: Ahmed Abu-Haniyeh MD, Internal Medicine Residency Program. Medicine Institute, Cleveland Clinic, Cleveland, USA, Tel+1 (216) 2720307; E-mail: abuhana@ccf.org

Received May 22, 2017; Accepted May 29, 2017; Published June 10, 2017

Citation: Abu-Haniyeh A, Alkukhun L, Al-Natour M, Hassan M, Tonelli A (2017) Arteriovenous Malformation in CLOVES Syndrome. Med Rep Case Stud 2: 135. doi: 10.4172/2572-5130.1000135

Copyright: $(2017$ Abu-Haniyeh A, et al. This is an open-access article distributed under the terms of the Creative Commons Attribution License, which permits unrestricted use, distribution, and reproduction in any medium, provided the original author and source are credited. 


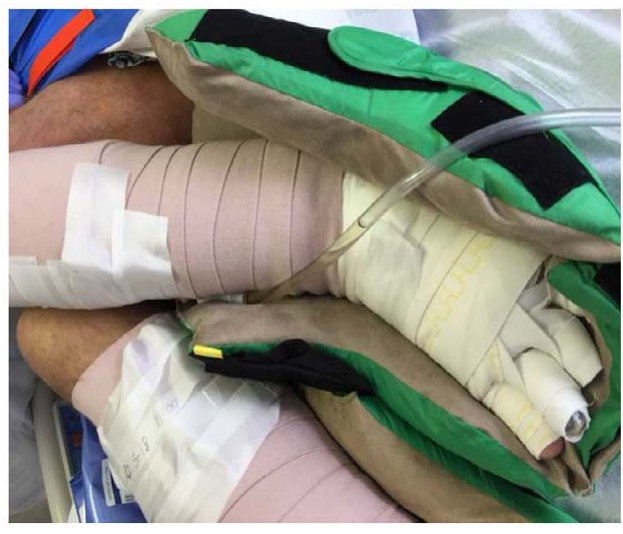

Figure 2: Lymphedema determination.

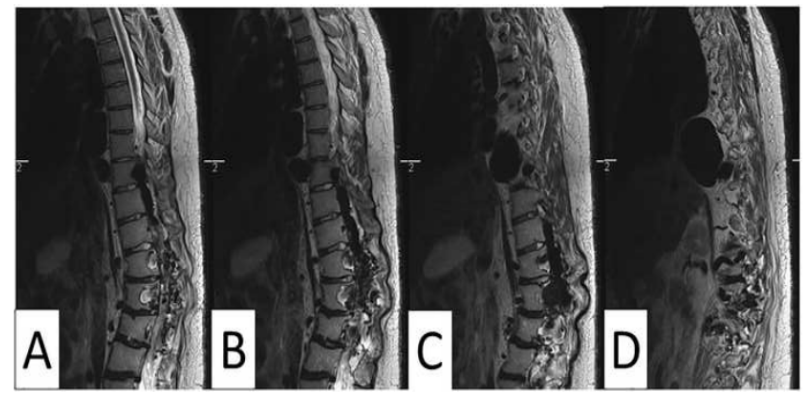

Figure 3: MRI spinal cord.

a left corneal transplant. Between the ages of 12-15 years old, he had progressive lower limbs weakness with a preference to crawl rather than walk. Radiographic imaging confirmed a right hip dislocation due to poor acetabular development. In addition, he was diagnosed with CLOVES syndrome after a spine magnetic resonance imaging (MRI) demonstrated an AVM compressing the spinal cord at T10-L4 levels. Interestingly, this AVM communicated with the azygous vein causing azygous phlebectasia (Figure 3). As an incidental finding, the brain MRI showed a mild descent of the cerebellar tonsils suggestive of Chiari malformation type I. The brain parenchyma had normal morphology and signal intensity, without intracranial mass, extra-axial fluid collection, or hydrocephalus. In addition, the craniocervical junction was normal in appearance. Imaging also showed left renal hypoplasia, double ureter and hepatosplenomegaly. At the age of 19 years old, he developed a spontaneous deep venous thrombosis in the setting of chronic moderate thrombocytopenia and a large size AVM. He was treated with long-term anticoagulation and received an inferior vena cava (IVC) filter. Embolization therapy of the AVM was performed on multiple occasions; nevertheless, the AVM's size increased. At the age of 26, the patient was admitted to our Medical Intensive Care Unit because of altered mental status, respiratory distress and circulatory shock. He was found to have pronounced thrombocytopenia and anemia, acute kidney injury and coagulopathy. He had prolongation of prothrombin and activated partial thromboplastin times, low plasma fibrinogen and elevation of $\mathrm{D}$ dimer and fibrin degradation products. The peripheral blood smear showed red blood cell fragments, helmet cells, polychromasia and thrombocytopenia suggestive of acute disseminated intravascular coagulation (DIC). Heparin platelet factor-4 antibodies were negative. During the hospitalization, he required intubation and treatment with vasopressors, fresh frozen plasma (FFP) and cryoprecipitates. Serial cultures, abdominal-pelvic computed tomography (CT), tagged white blood cells and echocardiogram were all negative for any source of infection. CT of the abdomen disclosed a massive thrombus at the level of the IVC filter, extending into the external and internal iliac veins, which was effectively treated with catheter directed thrombolysis. The patient's hemodynamics recovered. He was extubated and his hemoglobin and platelets levels stabilized. $\mathrm{He}$ was transferred afterwards to the regular nursing floor and eventually discharged to a skilled nursing facility.

\section{Discussion}

CLOVES syndrome manifestations can lead to severe complications. Scoliosis, skeletal discrepancy and fatty hypertrophy cause progressive deformities. Patients eventually suffer from joint dislocation and necrosis in the extremities due to vascular entrapment [13]. AVM increase the risk of pulmonary embolism [16-18] and if located in the paraspinal region may result in spinal cord compression [12]. Our patient had progressive lower extremities weakness and neurogenic bladder due a large AVM tethering the spinal cord. Unfortunately, the AVM's size and complexity at time of diagnosis limited the success of embolization therapy and the surgical option was waived given patient's comorbidities and high surgical risk. The Guidelines for the diagnosis and management disseminated intravascular coagulation mention a wide range of pathological conditions that can cause DIC including AVM [19]. AVM can induce consumptive coagulation by activating cytokines as part of a systemic inflammatory response or by causing the release of procoagulant substances $[19,20]$. AVM are lined by dysplastic cells exposed to stagnated blood, which can trigger chronic localized intravascular coagulation [21]. DIC has been reported in Klippel-Trenaunay patients; a syndrome of slowflow vascular malformations and skeletal and soft tissue hypertrophy $[22,23]$. Nevertheless, DIC triggered by AVM was never reported in CLOVES. It is unclear whether DIC in our patient was AVM-related or was due to a potential occult infection. CLOVES syndrome is rare, often misdiagnosed and difficult to manage. Patients eventually require procedures like debulking [13,14], amputations [13-15], resection of lipomas [14,16], spinal fixations [16] and AVM embolizations [12,14]. Definitive guidelines for management of this syndrome are still lacking [14]. Patients with CLOVES may develop encephalomalacia, hemimegalencephaly, ventriculomegaly, and bilateral pes equinovarus $[1,5,6,12]$. Developmental delay has also been reported [1]. Corneal keratoconus is a non-inflammatory thinning of the cornea that can be complicated with edema in the cornea resulting in corneal hydrops. This eventually leads to corneal scarring and vision impairment. The pathogenesis of this disorder remains unclear. Reported risk factors include eye rubbing in addition to genetic diseases affecting collagen and connective tissues. Our patient had corneal hydrops in both eyes in a span of less than two years. We do not know if Chiari malformation or corneal disease are associated with CLOVES. More research is needed to determine the significance of these findings. In addition, our patient was diagnosed with splenomegaly and double ureter. This is further evidence that visceral organs are affected in CLOVES $[1,13]$. Our patient was misdiagnosed as Klippel-Trenaunay syndrome at birth. This syndrome has characteristic findings that are similar to CLOVES like soft tissue hypertrophy and skeletal overgrowth. Patients also have capillary and venous malformations. However, our patient was found to have an AVM later in life which prompted the clinicians at to challenge his initial diagnosis. CLOVES syndrome is related to PIK3CA mutation; however, genetic confirmation was not pursued in our patient given vast clinical anifestations. In summary, CLOVES is 
rare syndrome but complications are common. In the case presented, we encountered a large AVM leading to spinal cord compression and coagulopathy. Our patient had progressive fatty tissue, skeletal overgrowth as well as double ureter and splenomegaly, supporting that CLOVES syndrome affects visceral organs. Other findings like Chiari malformation and corneal hydrops might also be related to the syndrome; however more evidence is needed to support these associations.

\section{References}

1. Sapp JC (2007) Newly delineated syndrome of congenital lipomatous overgrowth vascular malformations and epidermal nevi (CLOVE syndrome) in seven patients. Am J Med Genet 143: 2944-2958.

2. Alomari Al (2009) Characterization of a distinct syndrome that associates complex truncal overgrowth vascular and acral anomalies: a descriptive study of 18 cases of CLOVES syndrome. Clin Dysmorphol 18: 1-7.

3. Alomari Al (2009) A truly unusual overgrowth syndrome: an alternative diagnosis to Klippel-Trenaunay-Weber syndrome. Intern Med 48: 493-495.

4. Kurek KC (2012) Somatic mosaic activating mutations in PIK3CA cause CLOVES syndrome. Am J Hum Genet 90: 1108-1115.

5. Gucev ZS (2008) Congenital lipomatous overgrowth vascular malformations and epidermal nevi (CLOVE) syndrome: CNS malformations and seizures may be a component of this disorder. J Med Genet A 146: 2688-2690.

6. Alomari Al (2009) CLOVE(S) syndrome: expanding the acronym. J Med Genet 149: 295.

7. Harit D, Aggarwal A (2010) CLOVE syndrome with nevus unis lateris: report of a case Pediatr Dermatol. 27: 311-312.

8. Fernandez-Pineda I (2010) Perinatal clinical and imaging features of CLOVES syndrome. Pediatr Radiol 40: 1436-1439.

9. Sarici D (2014) A Neonate with CLOVES Syndrome Case Rep Pediat 84508474.
10. Puvabanditsin S (2014) Cloves syndrome: a case report and perinatal diagnostic findings. Genet Couns 25: 265-270

11. Gopal BSN, Keshava D, Selvaraj A (2015) Rare newly described overgrowth syndrome with vascular malformations-Cloves syndrome. Indian J Radio Imaging 25: 71-73.

12. Alomari Al (2011) Complex spinal-paraspinal fast-flow lesions in CLOVES syndrome: analysis of clinical and imaging findings in 6 patients AJNR. J Neuroradiol 32: 1812-1817.

13. Klein S (2012) Phenotypic progression of skeletal anomalies in CLOVES syndrome. J Med Genet 158: 1690-1695.

14. Ballieux $F$ (2013) Reconstructive surgery in the management of a patient with CLOVES syndrome. J Plast Reconstr Aesthet Surg 66: 1813-1815.

15. Bloom J, Upton J (2013) 3rd CLOVES syndrome. J Hand Surg 38: 2508-2512.

16. Alomari Al (2010) CLOVES syndrome with thoracic and central phlebectasia: increased risk of pulmonary embolism. J Thorac Cardiovasc Surg 140: 459463

17. Hedequist D (2015) Surgical Treatment of Spinal Deformity in Patients With CLOVES Syndrome: A Report of 4 Cases. J Pediatr Orthop 35: 682-686.

18. Kulungowski AM, SJ Fishman (2011) Management of combined vascular malformations. Clin Plast Surg 38: 107-120.

19. Levi M (2009) Guidelines for the diagnosis and management of disseminated intravascular coagulation British Committee for Standards in Haematology. J Haematol 145: 24-33.

20. Siegal T (1978) Clinical and laboratory aspects of disseminated intravascular coagulation (DIC): a study of 118 cases Thromb Haemost 39: 122-134.

21. Enjolras O (1997) Extensive pure venous malformations in the upper or lower limb: a review of 27 cases. J Acad Dermatol 36: 219-225.

22. Aronoff DM, M Roshon (1998) Severe hemorrhage complicating the KlippelTrenaunay-Weber syndrome. South Med J 91: 1073-1075.

23. Stein SR, JH Perlow, Sawai SK (2006) Klippel-Trenaunay-type syndrome in pregnancy. Obstet Gynecol Surv 61: 194-206. 